TECHNICAL NOTE

\author{
M. McDermott \\ B.F. Branstetter IV
}

\section{Percutaneous Drainage of Postoperative Laryngoceles}

SUMMARY: Laryngoceles are most frequently treated surgically, but if the patient's airway is compromised by mass effect, general anesthesia may be risky. We describe a technique for percutaneous drainage of postoperative laryngoceles and the outcome in a small series of patients.
T he laryngeal ventricle is an intralaryngeal space that lies between the false cords (superiorly) and the true cords (inferiorly). The saccule of the laryngeal ventricle is an extension of the ventricle superiorly along the inner surface of the thyroid cartilage. When the saccule becomes obstructed, it may expand and cause mass effect; this mass is called a "laryngocele." ${ }^{1}$ Laryngoceles may be air- or fluid-filled, and they have the potential to become infected. Laryngoceles are often asymptomatic, but symptoms such as neck swelling, hoarseness, dyspnea, and sleep apnea have been reported. The etiology of laryngoceles is varied, with both congenital and acquired factors playing a role. Posttherapy laryngoceles, however, are a well-recognized entity and may occur after surgery or radiation treatment. ${ }^{2}$ Surgical drainage and marsupialization are the treatments of choice for laryngoceles, either from an external approach or by endoscopic laser excision. ${ }^{3,4}$

Most laryngoceles are unilateral and, thus, do not threaten the patient's airway. However, laryngoceles may arise bilaterally in the posttreatment setting and may be a life-threatening complication of therapy. Surgical drainage of the laryngoceles in these patients may be difficult because of the tenuous airway. Anesthesia poses a risk of complete airway collapse, while emergent tracheostomy is a suboptimal treatment technique. Furthermore, in patients who have already undergone surgical or radiation treatment, the altered anatomy and likelihood of scarring in the surgical bed increase the risk of surgery.

In this report, we present a series of patients with bilateral postoperative laryngoceles who underwent percutaneous drainage in preparation for formal surgical treatment of the laryngoceles. Each of these patients underwent a supracricoid laryngectomy for laryngeal squamous cell carcinoma, including removal of the false cords. At the surgical margin, bilateral cystic masses formed due to fluid and mucus build-up in remnants of the laryngeal saccule. The patients had dramatic airway narrowing due to bilateral compression from the laryngoceles and were deemed to be at high surgical risk. A novel percutaneous drainage procedure was attempted and was successful in all 3 patients. We propose CT-guided percutaneous drainage as a safe and viable treatment option for patients with postoperative laryngoceles, either to improve the risk of surgery or as a definitive treatment.

Received February 10, 2010; accepted after revision February 15

From the Departments of Radiology (M.M., B.F.B.) and Otolaryngology (B.F.B.), University of Pittsburgh School of Medicine, Pittsburgh, Pennsylvania.

Please address correspondence to Barton Branstetter, MD, Department of Radiology, University of Pittsburgh School of Medicine, 200 Lothrop St, PUH Room D132, Pittsburgh,

PA 15213; e-mail: branstetterbf@msx.upmc.edu

DOI 10.3174/ajnr.A2095

\section{Technique}

Patients were selected for CT-guided percutaneous drainage on the basis of a history of supraglottic laryngectomy, clinical presentation of a narrowed glottic airway, and evidence of bilateral laryngoceles on CT. We have performed this procedure on 3 such patients. The minimum dimension of the patients' glottic airway before the procedure varied from 1 to $5 \mathrm{~mm}$. All 3 patients were men, with ages ranging from 48 to 65 years. Time since surgery ranged from 3 to 25 months, and no patient had endoscopic evidence of residual tumor in the portions of the upper aerodigestive tract that were accessible endoscopically. All 3 patients tolerated the procedure well, with substantial reduction in the radiologic size of the laryngoceles, and in all cases, the patient was rendered an appropriate candidate for formal surgical treatment of the laryngoceles.

The patient is positioned supine in the CT scanner, and a $2 \%$ lidocaine solution is used for a local anesthetic. Given the risk of airway collapse, conscious sedation or other intravenous anesthetics or analgesics should be avoided. Under CT fluoroscopic guidance, a 14-ga Tuohy needle is inserted from an anterolateral approach, avoiding the neurovascular sheath. In our experience, no dilator or introducer is required. Compared with the smaller bore needles used for fine-needle aspiration, a larger bore needle is necessary because of the high viscosity of the entrapped mucous in the laryngoceles. After CT confirmation that the first laryngocele has been adequately reduced, the other side is drained in the same fashion. The mucous drained from the laryngoceles should undergo cytologic examination to exclude recurrence of malignancy.

An otolaryngologist (or other physician familiar with placement of an emergency airway) must be present in the CT suite throughout the procedure in case an emergent airway becomes necessary. Postprocedural endoscopic evaluation of the larynx was available in the event that symptoms failed to improve or worsened during the procedure, but this was not required for any of our patients, so endoscopic evaluation was deferred until the patient's next scheduled clinical follow-up appointment.

\section{Case Presentation}

A 65-year-old man with squamous cell carcinoma of the larynx underwent definitive treatment with supraglottic laryngectomy with epiglottopexy. He presented 2 years after surgery with a high-pitched voice, progressive sleep apnea, and shortness of breath. Severe biphasic stridor was noted clinically. Contrast-enhanced neck CT showed symmetric cystic masses on the lateral sides of the remaining larynx, reducing the glottic airway to approximately $1 \mathrm{~mm}$ in diameter (Fig 1). The patient was considered by the anesthesiology team to be at very high risk of airway collapse during intubation, so the patient's otolaryngologists were uneasy about traditional surgical treatment of the lesions.

An otolaryngologist was invited to join the radiology team in the CT suite, in case an emergent airway was needed; then the patient was 


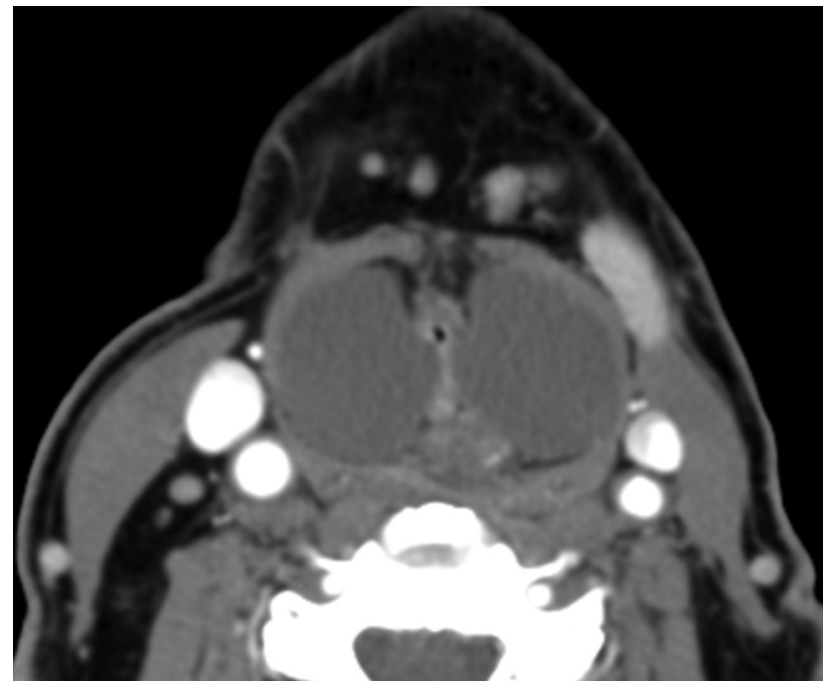

Fig 1. Postoperative laryngoceles. Preprocedural contrast-enhanced axial CT scan demonstrates bilateral internal laryngoceles compressing the glottic airway down to approximately $1 \mathrm{~mm}$.

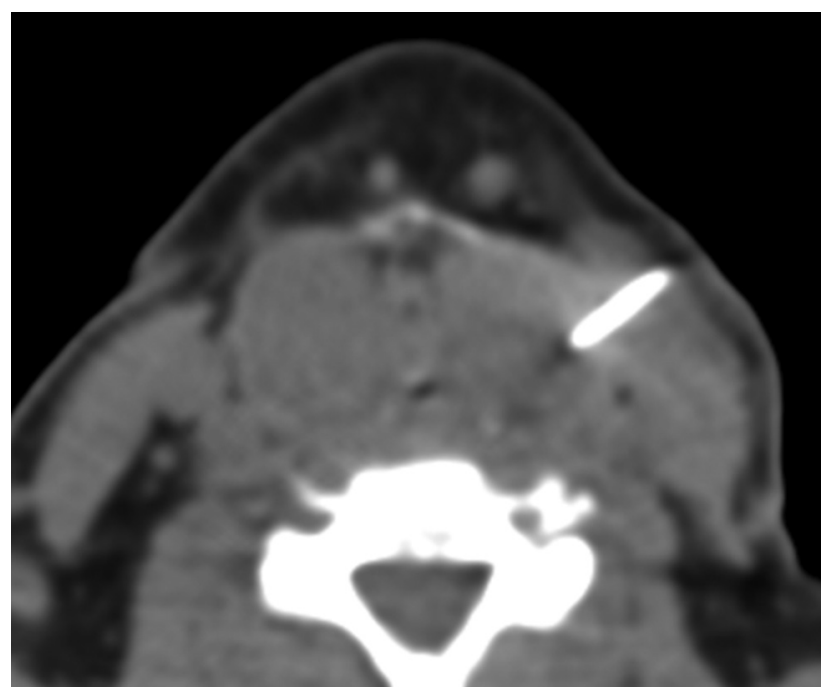

Fig 2. Percutaneous drainage of laryngoceles. Contrast-enhanced axial CT during the procedure shows a 14-ga needle inserted from an anterolateral approach into the left laryngocele.

positioned in the CT scanner and given a local anesthetic. Initially, a 25-ga spinal needle was inserted into the left laryngocele via an anterolateral approach. No material could be aspirated, so progressively larger spinal needles were inserted, until a 14-ga Tuohy needle from a lumbar drain kit was needed, at which point $15 \mathrm{~mL}$ of thick yellow mucous was drained (Fig 2). (When the procedure was performed on other patients, we began with the 14-ga needle; it required no introducer or dilator.) After drainage of the first laryngocele, the patient had complete resolution of voice changes as well as shortness of breath and stridor, and in fact, the patient immediately fell asleep in the CT scanner due to chronic fatigue from sleep apnea. The rightsided lesion was then drained uneventfully. Radiologic follow-up 2 weeks after the procedure showed partial drainage of both laryngoceles, with an improved epiglottic and glottic airway (Fig 3).

The original plan for this patient called for definitive surgical treatment of the laryngoceles, but his clinical improvement was so dramatic that he refused immediate surgery. He was asymptomatic for 18 months after the procedure but then had recurrence of clinical

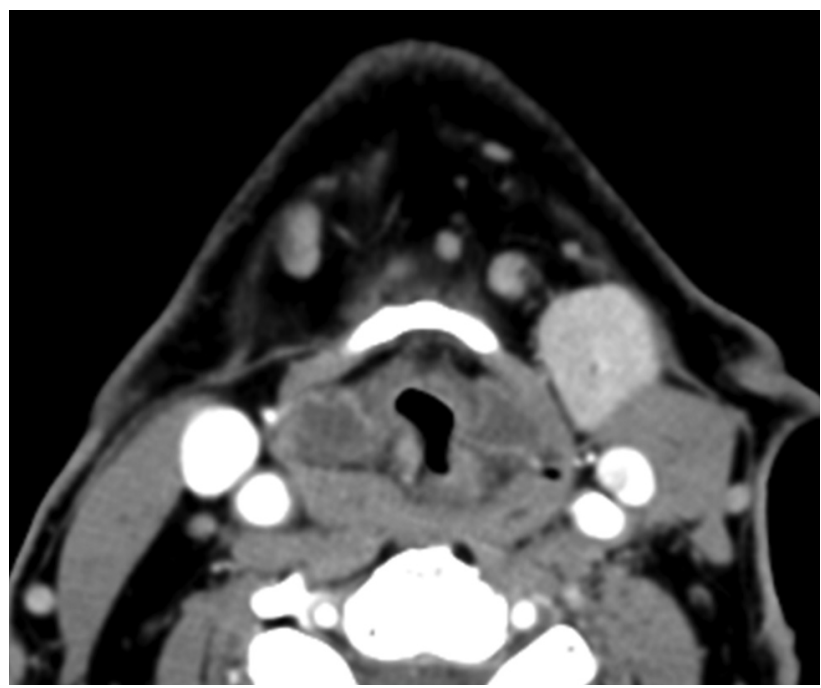

Fig 3. Contrast-enhanced axial CT 2 weeks after the procedure shows reduced bilateral laryngoceles. Note the redundant folds of the laryngoceles and the expanded glottic airway.

symptoms. The patient is now considering whether to undergo repeat percutaneous drainage or definitive surgery.

\section{Discussion}

Laryngoceles are uncommon, with an estimated incidence of 1 per 2.5 million people per year. ${ }^{5}$ Laryngoceles are unilateral $85 \%$ of the time, so bilateral laryngoceles are an especially rare occurrence. ${ }^{1}$ There is a strong male preponderance, with rates in men 5-7 times greater than rates in women.

Laryngoceles are classified as internal, external, or mixed. Internal laryngoceles stay within the confines of the thyrohyoid membrane, while external laryngoceles extend beyond the cartilaginous boundaries of the larynx and maintain only a thin connection to their site of origin. Combined or mixed laryngoceles have substantial components both inside and outside the larynx.

The term laryngocele is generally used if the mass is airfilled or has an air-fluid level demonstrating an open communication with the laryngeal lumen. The term "saccular cyst" is used by some authors to refer to masses that are completely filled with fluid or mucous and thus presumably lack such a communication. Other authors use the term laryngocele regardless of the contents of the lesion. ${ }^{3,4}$

The use of the term "laryngocele" in reference to patients who have undergone supraglottic laryngectomy is somewhat problematic, in that the upper surface of the laryngeal ventricle has been surgically removed. Thus, these lesions do not form in the same manner as most laryngoceles. Nevertheless, the radiographic appearance of the lesions and their histologic contents are the same as those of other laryngoceles, so we have continued the practice of applying this term. Some authors may prefer the more general term "mucocele," but this term is also problematic because it is usually applied to enlarged air cells.

In our limited series, CT-guided percutaneous drainage of postoperative laryngoceles is a safe and effective procedure. On the basis of our experience, this procedure can act as a bridge to more definitive surgical treatment and safely secures an otherwise dangerously narrow airway, so that in the future, 
direct laryngoscopy and general anesthesia can be administered with less risk of airway collapse. The procedure is also effective enough at relieving airway compression that it can stand alone as a treatment in patients in whom surgery would be unadvisable. However, we do not have enough clinical evidence and follow-up to speak to the rate of recurrence or whether percutaneous drainage will be effective as a sole treatment technique in the long term.

Another advantage of percutaneous drainage is that it acts as a diagnostic as well as a therapeutic procedure. The drained mucous can undergo cytologic analysis to test for recurrent malignancy, which is a concern in any patient with a history of laryngeal squamous cell carcinoma. In particular, there should be a high level of concern in patients who develop a laryngocele years after therapy and in those in whom a concomitant enhancing mass is seen on CT.
In conclusion, CT-guided percutaneous drainage of postoperative laryngoceles can be a safe and effective procedure, both as a definitive treatment and as a means to secure the patient's airway before surgical excision, even in the setting of severe airway compromise.

\section{References}

1. Pennings RJ, van den Hoogen FJ, Marres HA. Giant laryngoceles: a cause of upper airway obstruction. Eur Arch Otorhinolaryngol 2001;258:137-40

2. Stocker D, Kelly W, Fitzpatrick T, et al. Bilateral external laryngoceles following radioiodine ablation for Graves disease. Arch Intern Med. 2002;162: 2007-09

3. Devesa PM, Ghufoor K, Lloyd S, et al. Endoscopic $\mathrm{CO}_{2}$ laser management of laryngocele. Laryngoscope 2002;112:1426-30

4. Thabet MH, Kotob H. Lateral saccular cysts of the larynx: aetiology, diagnosis and management. J Laryngol Otol: 2001;115:293-97

5. Dursen G, Ozgursoy OB, Beton $\mathrm{H}$, et al. Current diagnosis and treatment of laryngocele in adults. Otolaryngol Head Neck Surg 2007;136:211-15 